\title{
EFFECT OF STIMULI IN RIGHT HEMISPACE ON LEFT-SIDED NEGLECT IN A LINE CANCELLATION TASK
}

\author{
Charles M. Butter* \\ Department of Psychology, The University of Michigan, and Department of Physical Medicine and \\ Rehabilitation, The University of Michigan Medical Center
}

(Received 3 May 1991; accepted 4 June 1992)

\begin{abstract}
Twenty-seven patients with right cercbral infarets resulting in left-sided neglect in a line cancellation task were also tested for line cancellation when the lines were confined to the left half of sheets. All but one patient (whose neglect in the former condition was minimal) were still impaired in the latter condition, a finding that indicates that hyperattraction to test stimuli in the unneglected (right) hemispace was not the crucial factor preventing them from completing the standard version of the cancellation task, although it may have played some role. The results are consistent with the conclusion that hyperattention to stimuli in the unneglected hemificld, and possibly to stimuli in the unneglected hemispace, together with other factors, could account for the patients' impairment in line cancellation.
\end{abstract}

\section{INTRODUCTION}

UNILA IERAL VISUAL NEGLECT is a disorder that often is present after damage to the right hemisphere; it is marked by an impairment in detecting or responding to visual stimuli in contralateral visual field or hemispace and in exploring the contralateral side of visual space. Patients with left-sided visual neglect frequently are impaired in finding visual targets on the left side of displays; for example, in a line cancellation task, they often fail to mark lines on the left side of a sheet [7].

One factor that may be responsible for unilateral neglect in search tasks is a deficiency in shifting attention and/or performing orienting or manual movements to the neglected side. Patients with unilateral parietal lesions, (which frequently result in unilateral neglect) have difficulty disengaging their attention from a stimulus in order to reorient it in the contralesional direction $[11,12]$. Furthermore, attention-attracting stimuli on the neglected side reduce line-bisection errors of neglect patients [2]. Evidence for a selective impairment in executing movements toward the neglected side, i.c. unilateral directional hypokincsia, has also been presented (e.g. Refs [5], [6] and [10]).

Another factor that may be responsible for unilateral neglect is hyperattention or a motor bias to the unneglected side. Stimuli ipsilateral to the lesion have a "magnetic" effect on the attention of neglect patients [13]. The frequent occurrence of gaze shifts to the unneglected side in patients with unilateral neglect is also consistent with this view [4]. On the basis of these and other findings, KINSBOURNE [8] has suggested that neglect is due to release of the intact hemisphere's orienting mechanisms from the inhibition normally exerted by the damaged hemisphere.

\footnotetext{
*Address for reprints: Neuroscience Laboratory, 1103 East Huron St., Ann Arbor, MI 48109, U.S.A.
} 
There is recent evidence that hyperattention to unneglected stimuli (i.e. "magnet effect") is an important determinant of neglect in a cancellation task. When patients with left-sided neglect in a line-marking task erase the lines, they show much less neglect than they do when marking the lines [9]. In fact, one-half of the patients who showed left-sided neglect when marking lines performed errorlessly when erasing them. The investigators who reported this finding concluded that "hyperattention" to stimuli in right hemispace is a determinant of leftsided neglect [9].

However, it is possible that hyperattention to stimuli in the right hemifield also may be a crucial factor in neglect of left-sided lines. Patients with left-sided neglect, unlike normal subjects, typically begin cancelling stimuli on the far right side and then proceed leftwards. Thus, the stimuli that they already cancelled tend to lie in their right hemifields (assuming they foveate stimuli as they cancel them). It is possible that hyperattraction to stimuli in the right hemifield as opposed to those in right hemispace can be separated by presenting neglect patients with two versions of a cancellation task - one in which the right half of the sheet is blank, the other in which items to be cancelled occupy both halves. One would expect that in the former task, patients with left-sided neglect would first be attracted to the rightmost lines and proceed leftwards. If a magnet effect of right-field stimuli is responsible in part or in whole for their cancellation deficit, they should cancel as many lines as they do when presented with a sheet filled with lines and then stop. If, on the other hand, the magnet effect demonstrated by MARK et al. [9] is hemispace-specific, and if this is the only factor interfering with the performance of a significant proportion of neglect patients as their findings suggest, then one would expect that in a large sample of patients showing neglect in a standard (full-sheet) cancellation task, a significant proportion of them should not be impaired when the stimuli are restricted to the left side of sheets on which they are presented. The present experiment tested these predictions.

\section{METHODS}

\section{Patients.}

Twenty-seven patients, 12 men and 15 women, in several hospitals (Veterans' Administration Medical Center and Shands Hospital, Gainesville, Florida; University of Michigan Medical Center and Veterans' Administration Hospital, Ann Arbor, Michigan; Rehabilitation Hospital, Detroit, Michigan) served as subjects (see Table 1). All were selected for left-sided neglect in the line-cancellation test described below. Twenty-five of them had sustained cerebral vascular accidents in the distribution of the right middle cercbral artery detected in CT scans. The remaining two (Nos 11 and 18) underwent evacuation of hematomas that resulted in infarcts, also detected in CT scans, involving the right hemisphere. Time from the onset of symptoms or, in the case of evacuated hematomas, from surgery, to the beginning of testing was not less than 13 days. Other infonntion concerning the patients" characteristics are shown in Table 1. Unless otherwise indicated, the patients were right-handed by self-report.

In the screening test, patients were presented with a single sheet, $28.3 \mathrm{~cm}$ in width and $21.7 \mathrm{~cm}$ in height, on which 60 lines, $1.7 \mathrm{~cm}$ in length, were shown in varying orientations. An equal number of lines were located on the left and right sides of the sheet, which was centered with respect to the patient's body axis; care was taken to insure that patients did not move their bodies during testing. The patients were asked to mark all of the lines with a pencil held in the right hand, and to put down the pencil when they completed the task. Left-sided neglect was defined by failure to cancel more lines on the left side than on the right side of the page. All patients were also tested for (a) visual fields by confrontation with a moving finger, (b) gaze deviation, and (c) saccadic and pursuit eye movements. Patients without visual field defects were tested by confrontation for visual extinction. In addition to the line cancellation test used to screen patients for neglect, several other tests of unilateral neglect (clock construction, line bisection, copying a drawing and reading), the details of which are presented elsewhere [2], were also administered.

\section{Testing}

The patients were tested in a single session three times with a full sheet of lines identical to those presented in the screening test described above (whole-sheet test), and with three sheets identical to these, except that the right halves were blank (right-half blank tests). The whole sheet and right-half blank sheets werc presented alternately. Thirtcen 
Table 1. Characteristics of patients

\begin{tabular}{|c|c|c|c|c|}
\hline Group & Pt. & Age/Sex & Lesion* & $\begin{array}{l}\text { Time from } \\
\text { onset of } \\
\text { symptoms to } \\
\text { testing } \\
\text { (days) }\end{array}$ \\
\hline \multirow[t]{13}{*}{$1 \dagger$} & 1 & $64 / \mathrm{m}$ & CVA P-T & 21 \\
\hline & 2 & $74 / \mathrm{m}$ & CVA P-T-O & 17 \\
\hline & 3 & $69 / \mathrm{m}$ & CVA O-T & 18 \\
\hline & 4 & $71 / \mathrm{m}$ & CVA P-T-F & 36 \\
\hline & 5 & $71 / \mathrm{m}$ & CVA P-F & 20 \\
\hline & 6 & $77 / \mathrm{m}$ & CVA T-O & 21 \\
\hline & 7 & $59 / \mathrm{f}$ & CVA P-T & 13 \\
\hline & 8 & $72 / \mathrm{f}$ & CVA P & 32 \\
\hline & 9 & $73 / 1$ & CVA P-I-H-O & 50 \\
\hline & 10 & $68 / f$ & CVA P-T-F & 52 \\
\hline & 11 & $59 / \mathrm{f}$ & P-F hematoma evac. & 17 \\
\hline & 12 & $71 / \mathrm{f}$ & CVA P-T-O & 23 \\
\hline & 13 & $67 / \mathrm{f}$ & CVA P & 36 \\
\hline \multirow[t]{14}{*}{$2 \ddagger$} & 14 & $58 / \mathrm{m}$ & CVA F-T & 39 \\
\hline & 15 & $64 / \mathrm{m}$ & CVA P & 46 \\
\hline & 16 & $59 / \mathrm{m}$ & CVA F-P & 26 \\
\hline & 17 & $60 / \mathrm{m}$ & CVA F-T-P & 6 years \\
\hline & 18 & $68 / \mathrm{IIl}$ & T-F hematonla evac. & 27 \\
\hline & 19 & $87 / \mathrm{m}$ & CVA F-P & 12 \\
\hline & $20 \S$ & $55 / \mathrm{f}$ & CVA T-P-F & 229 \\
\hline & 21 & $59 / \mathrm{f}$ & CVA T-F & 19 \\
\hline & 22 & $84 / f$ & CVA T-P & 26 \\
\hline & 23 & $73 / \mathrm{f}$ & CVA F-T & 17 \\
\hline & 24 & $56 / \mathrm{f}$ & CVA T-P & 18 \\
\hline & 25 & $74 / f$ & CVA F-T-P & 63 \\
\hline & 26 & $56 / f$ & CVA P-O & 12 \\
\hline & 27 & $72 / \mathrm{f}$ & CVA T-P & 26 \\
\hline
\end{tabular}

${ }^{*} \mathrm{P}=$ parietal; $\mathrm{O}=$ occipital; $\mathrm{T}=$ temporal; $\mathrm{F}=$ frontal; all lesions in right hemisphere.

f Patients who cancelled lines only on the right side of sheets.

$\ddagger$ Patients who cancelled lines on both sides of sheet.

§Left-handed.

patients were tested first with a whole sheet; the remainder were tested first with a right-hand blank sheet first. Several of the patients did not complete testing. Patients 1, 5,6 and 20 were tested with two sheets in each condition; two patients (Nos 7 and 11 ) were tested with one sheet in each condition.

\section{RESULTS $\Lambda$ ND DISCUSSION}

All the patients began both tests by cancelling the rightmost lines and then proceeding to the left. Thirteen of the 27 patients (Group 1) cancelled lines only on the right side in the whole-sheet test (see Table 2). All but one of them (No. 1) cancelled some lines on the left when the right side was blank. However, none of the Group 1 patients marked all 30 lines on the left side in the right-side blank test; on average, they marked only $33 \%$ of these lines, and the most marked was $73 \%$. Furthermore, the 12 patients in Group 1 who cancelled some lines in the right-hand blank test cancelled significantly less lines in this condition than they did in the whole-sheet condition $(t=4.873$; d.f. $=11 ; P<0.001)$. A significant correlation $(r=+0.790 ; P=0.002)$ was found between the degree to which the patients in Group 1 were impaired in the whole-sheet test (assessed by the number of lines they failed to cancel in this 
Table 2. Performance of neglect patients in right-half blank (RHB) and whole-sheet (WS) line-cancellation tests

\begin{tabular}{|c|c|c|c|c|c|}
\hline \multirow[b]{2}{*}{ Group } & \multirow[b]{2}{*}{$N$} & \multirow{2}{*}{$\begin{array}{c}\text { No. showing } \\
\text { magnet } \\
\text { effect }\end{array}$} & \multicolumn{3}{|c|}{$\begin{array}{c}\text { Mean } \\
\text { No. of lines cancelled }(\mathrm{SD})^{*}\end{array}$} \\
\hline & & & $\begin{array}{c}\mathrm{RHB} \\
\text { tesi }\end{array}$ & $\begin{array}{l}\text { WS test } \\
\text { (right side) }\end{array}$ & $\begin{array}{l}\text { WS test } \\
\text { (left side) }\end{array}$ \\
\hline $1 \dagger$ & 13 & $12(91.7 \%)$ & $\begin{array}{l}10.7 \\
(4.9)\end{array}$ & $\begin{array}{l}15.8 \\
(5.8)\end{array}$ & \\
\hline $2+$ & 14 & $13(92.9 \%)$ & $\begin{array}{l}21.1 \\
(6.8)\end{array}$ & $\begin{array}{l}30.0 \\
(0.0)\end{array}$ & $\begin{array}{l}13.7 \\
\{8.8)\end{array}$ \\
\hline
\end{tabular}

* Maximum $=30$ on each side.

† Cancelled only lines on right side in whole-sheet tests.

${ }_{+}^{+}$Cancelled lines on both sides in whole-sheet tests.

condition) and the degree to which they benefitted from deleting lines from the right side of the sheets (i.e. the difference between the number of lines cancelled in the two tests). That is, as the severity of the Group 1 patients' impairment increased, the degree of benefit from eliminating lines on the right increased.

The remainder of the patients (14/27), those comprising Group 2, marked some lines on the left side in the whole-sheet condition. In the right-side blank condition, only one of these patients (No. 20) marked all the lines on the left side; this patient cancelled all but one line on the left side in the whole-sheet test. On the average, the Group 2 patients cancelled $67 \%$ of the lines in the right-side blank test (see Table 2); two-thirds of them cancelled less than $90 \%$ of the lines in this condition. Nevertheless, the Group 2 patients cancelled significantly more lines on the left side in the right-side blank test than they did in the whole-sheet test $(t=3.705$; d.f. $=12 ; P<0.025$ ).

There was a significantly larger proportion of patients with hemianopia in Group 1 than in Group $2\left(\chi^{2}=4.805 ; P<0.05\right)$. Whereas the two groups did not differ in frequency of gaze deviation, disorders in saccadic and pursuit movements were found significantly more often in Group 1 than in Group $2\left(\chi^{2}=3.117, P<0.10\right)$. All Group 1 patients showed neglect in tests other than line cancellation, whereas the Group 2 patients showed neglect less frequently. In two of these tests (clock construction and line bisection) the group differences were significant (for clock construction, $\chi^{2}=8.128, P<0.01$; for line bisection, $\chi^{2}=5.653$, $P<0.02$ ). Thus, it seems likely that membership in Groups 1 and 2 reflect true differences in the severity of neglect.

If the finding [9] that many patients no longer neglect lines when they erase rather than mark them was due to eliminating a magnet effect of lines in the unneglected hemispace, then one would expect that some of the patients in the present study would no longer neglect lines limited to the left half of the sheets. The only patient who was unimpaired in this condition was minimally impaired in the whole-sheet test, where she ignored only one line, which was located on the lower left-side. In fact, the patients in Group I consistently cancelled somewhat fewer lines in the right-half blank test than they did on the right side in the whole sheet test. It is possible that differences between our line cancellation task and that of Mark et al. [9] in factors such as the length, number or density of lines may account for our failure to find a significant proportion of neglect patients who showed no impairment when lines were deleted from the right side of the sheets. Thus, the conclusion that a hemispace effect was absent or weak in this experiment does not necessarily imply that it was absent in the 
experiment of MARK et al. [9]. It might be argued that the patients failed to show the degree of benefit in the right-half blank condition expected by the right hemispace hyperattraction hypothesis because in this condition their attention was attracted to extra-task objects in right hemispace or to the right edge of the sheet. However, the same kinds of stimuli were also present in the study of Mark et al. and did not prevent one-half of their patients from showing normal cancellation performance when they erased lines. Why, then, should these stimuli prevent a significant (or even small) proportion of the 27 patients in the present study from showing normal performance in the right-half blank condition?

It is possible that the patients in the present study did not benefit as expected by the right hemispace hyperattention hypothesis because they differed from the patients of Mark et al. in the degree of attention directed to stimuli in right hemispace. Had they been tested with the procedure of Mark et al., they might not have shown the degree of benefit of erasing lines that their patients showed. While this possibility exists, it is not a likely one, for the patients in the two studies had similar lesions due to stroke and were selected for neglect by similar criteria.

The region within which Group 1 patients cancelled lines in the right-half blank condition was remarkably similar in shape to the region where they cancelled lines on the right side in the whole-sheet condition. This observation supports the view that their deficit was due to a hemifield magnet effect. However, the present findings cannot be accounted for solely by a hemifield magnet effect. If that were the case, then (a) patients in Group 1 would have cancelled as many lines in the right-half blank test as they did in the whole-sheet test, and (b) Group 2 patients would have cancelled all the lines in the right-half blank test. Since the patients consistently cancelled somewhat fewer lines than would be expected if a hemifield magnet effect were wholly responsible for their deficit, it would appear that some other factor or factors were in addition responsible for their impairment in the whole-sheet condition. Among these possible factors are deficient orientation to the neglected side [3] and hemihypokinesia [5], which might explain why the Group 1 patients cancelled fewer lines (on the left side) in the right-half blank condition than they did on the right side in the wholesheet condition. It should be noted that since the patients were free to move their heads as well as their eyes leftward as they cancelled, a head-centered spatial effect may have played a role in their neglect.

Acknowledgements-The author thanks Dr Kenneth M. Heilman (Dept. of Neurology, University of Florida, School of Medicine). Dr Fred Lamb (Rehabilitation Institute, Detroit), Dr Michael Welch and Kara L. Dietrich, R.N. (I Ienry Ford I Iospital, Detroit), and Dr Michael Welch (I Ienry Ford I Iospital, Detroit) for making it possible to test patients in their services. The author also thanks Alfreda Onimo for her editorial assistance. The research reported here was supported by National Research Service Award from the National Institute of Neurological and Communicative Disorders and Stroke.

\section{REFERENCES}

1. Bisiach, E., Capitani, E. and Porta. E. Two basic properties of space representation in the brain: evidence from unilateral neglect. J. Neurol. Neurusury. Psychiat. 48, 141-144, 1985.

2. Butter, C. M., KIRSCH, N. and ReEves. G. The effect of lateralized dynamic stimuli on unilateral spatial neglect following right hemisphere lesions. Restor. Neurol. Neurosci. 2, 39-46, 1990.

3. Butter, C. M., Mark, V.W. and Heilman, K. M. An experimental analysis of factors underlying neglect in line bisection. J. Neurol. Neurosurg. Psychiat. 51, 1581-1583, 1988.

4. DeRenzi, E., Colombo, A., Faglioni, P. and Gibertoni, M. Conjugate gaze paresis in stroke patients with unilateral damage. Arch. Neurol. 39, 482-486, 1982.

5. Heilman, K. M., Bowers, D., Coslett, H. B., When, H. and Watson, R. T. Directional hypokinesia: 
prolonged reaction times for leftward movements in patients with right hemisphere lesions and neglect. Neurology 35, 855-859, 1985.

6. Heilman, K. M., Bowers, D. and Watson, R. T. Performance on hemispatial pointing task by patients with reglect syndrome. Neurology 33, 661 664, 1983.

7. Heilman, K. M., Watson, R. T. and Valenstein, E. Neglect and related disorders. In Clinical Neuropsychology, K. M. Heilman and E. Valenstein (Editors), pp. 243-294. Oxford University Press, New York, 1985.

8. Kinsbourne, M. Mechanisms of unilateral neglect. Neurophysiological and Neuropsychological Aspects of Spatial Neglect, M. JEANNEROD (Editor), pp. 69 86. Elsevier, New York, 1987.

9. Mark, V. S., Kooistra, C. A. and Heilman, K. M. Hemispatial neglect affected by non-neglected stimuli. Neurology 38, 1207-1211, 1988.

10. Meador, K. J., Watson, R. T., Bowers, D. and Heilman, K. M. Hypometria with hemispatial and limb motor neglect. Brain 109, 293-305, 1986.

11. Posner, M. I., Walker, J. A., Friedrich, F. F. and Rafal, R. D. Effects of parietal injury of covert orienting of attention. J. Neurosci. 4, 1863-1874, 1984.

12. Posner, M. I., Walker, J. A., Friedrich, F. F. and Rafal, R. D. How do the parietal lobes direct covert attention? Neuropsychologia 25, 135-146, 1987.

13. Silber pfenNig, J. Contributions to the problems of eye movements. Disturbances of ocular movements with pseudohemianopia in frontal lobe tumors. Confin. Neurol. 4, 1 13, 1941. 